\title{
Nonlinear Tunneling of Surface Plasmon Polaritons in Periodic Structures Containing Left-Handed Metamaterial Layers
}

\author{
Munazza Zulfiqar Ali \\ Physics Department, Punjab University, Quaid-i-Azam Campus, Lahore 54590, Pakistan \\ Correspondence should be addressed to Munazza Zulfiqar Ali; munazzazulfiqar@yahoo.com
}

Received 3 September 2015; Accepted 30 November 2015

Academic Editor: Rosa Lukaszew

Copyright ( 2015 Munazza Zulfiqar Ali. This is an open access article distributed under the Creative Commons Attribution License, which permits unrestricted use, distribution, and reproduction in any medium, provided the original work is properly cited.

\begin{abstract}
The transmission of surface plasmon polaritons through a one-dimensional periodic structure is considered theoretically by using the transfer matrix approach. The periodic structure is assumed to have alternate left-handed metamaterial and dielectric layers. Both transverse electric and transverse magnetic modes of surface plasmon polaritons exist in this structure. It is found that, for nonlinear wave propagation, tunneling structures are formed to transform nontransmitting frequencies into transmitting frequencies and hence transmission bistability is observed. It is further observed that the structure shows sensitivity with respect to the polarization of the electromagnetic field for this phenomenon.
\end{abstract}

\section{Introduction}

As soon as metamaterials touched the optical regime, they have started to interfere with the field of plasmonics. Plasmonics is concerned with the unique properties and applications of surface plasmon polaritons (SPPs), that is, electromagnetic excitations that exist at interface between two media and decay exponentially away from the interface. Conventionally SPPs are studied at the interface between metal and dielectric due to strong coupling between light and free electrons of metal [1-7]. The main interest in SPPs arises due to its unique properties such as its ability to overcome the diffraction limit at the nanometer scale, subwavelength confinement, and strong field enhancement. The fundamental and applied research in this field has grown tremendously because of its promising applications in optics, data storage, sensors, and so forth [8-12]. Recent studies have revealed the existence of SPPs at the interface between dielectric and lefthanded metamaterials (LHM) [13-17]. Left-handed metamaterials are artificial composites having simultaneous negative value of electric permittivity and magnetic permeability in a certain frequency range [18-22]. These structures have been experimentally realized in microwave and infrared range $[23,24]$. The main advantage of studying SPPs at the LHMdielectric interface is the fact that both the transverse electric
(TE) and transvers magnetic (TM) modes can exist here. The existence and tunneling of SPPs in different type of periodic structures is also being studied $[5,25]$. Models are under consideration to transfer these states along specific structures $[26,27]$. Future photonics exploiting the properties of surface plasmon polaritons will require nonlinear components such as switching devices. Optical switches are based on optical bistability and play an important role in nonlinear optics. Different theoretical models in micro- and nanoscales for bistable behavior of propagating waves have been studied previously $[28,29]$. Now new avenues are being explored by combining optically nonlinear materials with plasmonic materials $[30,31]$, and still this is an area that needs more attention.

Here we are concerned with the nonlinear tunneling phenomenon of SPPs in a periodic structure composed of alternate left-handed metamaterial and dielectric layers. Both the TE and TM modes are studied here. By using the transfer matrix approach $[32,33]$, the transmission coefficient of the structure is calculated and electric field profiles within the structure are plotted. It is observed that if one of the layers of the periodic structure is considered to be nonlinear, the decaying states become the tunneling states and the phenomenon of optical bistability can be observed. This phenomenon is broadly used in optical storage, optotransistors, 


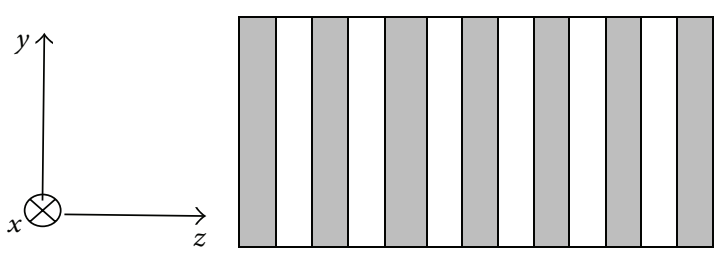

Figure 1: The schematic diagram of the structure under consideration; shaded layers represent the LH layers whereas white layers represent $\mathrm{RH}$ layers.

resonators, and all optical switches. This phenomenon has recently been studied in a special configuration for surface plasmons polaritons [34].

\section{Mathematical Model}

We consider a periodic structure containing alternate lefthanded metamaterial (LHM) and dielectric layers (these are termed here as right-handed material (RHM) layers) as schematically shown in Figure 1. The left-handed metamaterials are inherently lossy and dispersive. The electric permittivity " $\varepsilon$ " and magnetic permeability " $\mu$ " of the lefthanded metamaterial can be described by the Drude model $[21,22]$. The structure parameters are defined as follows:

For LHM layer,

$$
\begin{aligned}
& \varepsilon_{1}=1-\frac{\omega_{\mathrm{pe}}^{2}}{\omega^{2}+i \gamma \omega}, \\
& \mu_{1}=1-\frac{\omega_{\mathrm{pm}}^{2}}{\omega^{2}+i \gamma \omega} .
\end{aligned}
$$

For RHM layer,

$$
\begin{aligned}
\varepsilon_{2, \mathrm{nl}} & =\varepsilon_{2}+\alpha I, \\
\mu_{2} & =1 .
\end{aligned}
$$

In the above expressions, $\omega$ is the frequency of incident radiation, $\omega_{\mathrm{pe}}$ and $\omega_{\mathrm{pm}}$ are the plasma electric and plasma magnetic frequencies, $\gamma$ is the damping coefficient, $\alpha$ is the nonlinear Kerr coefficient, and $I$ is the intensity of incident radiation.

The electric and magnetic fields in the given geometry (Figure 1) can be written as follows:

For TM mode,

$$
\begin{aligned}
H & =\left(0, H_{y}, 0\right) \exp (i \vec{k}-i \omega t), \\
E & =\left(E_{x}, 0, E_{z}\right) \exp (i \vec{k}-i \omega t) .
\end{aligned}
$$

For TE mode,

$$
\begin{aligned}
H & =\left(H_{x} 0, H_{z}\right) \exp (i \vec{k}-i \omega t), \\
E & =\left(0, E_{y}, 0\right) \exp (i \vec{k}-i \omega t) .
\end{aligned}
$$

We are looking for solutions that are localized at the interfaces and decay away from the interfaces, so

$$
k=k_{x}+i k_{z}
$$

where

$$
\omega^{2}=\frac{c^{2}}{n_{j}^{2}}\left(k_{x}^{2}-k_{z j}^{2}\right) \quad j=1,2 .
$$

In the above expressions $k_{x}$ and $k_{z}$ are assumed to be real and positive.

The condition for the existence of SPP can be written as follows [33]:

For TM mode,

$$
\frac{k_{z 1}}{\varepsilon_{1}}+\frac{k_{z 2}}{\varepsilon_{2}}=0 .
$$

For TE mode,

$$
\frac{k_{z 1}}{\mu_{1}}+\frac{k_{z 2}}{\mu_{2}}=0 .
$$

Using (4) and (5a) and (5b), one has the following:

For TM mode,

$$
\begin{aligned}
k_{x}^{2} & =k_{\mathrm{SSP}}^{2}=\frac{\left(\omega^{2} / c^{2}\right) \varepsilon_{1} \mu_{1}\left(1-\varepsilon_{1} \mu_{2} / \varepsilon_{2} \mu_{1}\right)}{\left(1-\varepsilon_{1}^{2} / \varepsilon_{2}^{2}\right)} \\
& =\frac{\left(\omega^{2} / c^{2}\right) \varepsilon_{2} \mu_{2}\left(\varepsilon_{2} \mu_{1} / \varepsilon_{1} \mu_{2}-1\right)}{\left(\varepsilon_{2}^{2} / \varepsilon_{1}^{2}-1\right)} .
\end{aligned}
$$

For TE mode,

$$
\begin{aligned}
k_{x}^{2} & =k_{\mathrm{SSP}}^{2}=\frac{\left(\omega^{2} / c^{2}\right) \varepsilon_{1} \mu_{1}\left(1-\varepsilon_{2} \mu_{1} / \varepsilon_{1} \mu_{2}\right)}{\left(1-\mu_{1}^{2} / \mu_{2}^{2}\right)} \\
& =\frac{\left(\omega^{2} / c^{2}\right) \varepsilon_{2} \mu_{2}\left(\varepsilon_{1} \mu_{2} / \varepsilon_{2} \mu_{1}-1\right)}{\left(\mu_{2}^{2} / \mu_{1}^{2}-1\right)} .
\end{aligned}
$$

The value of normal component $k_{z j}$ is obtained by substituting $k_{x}$ from (6a) and (6b) into (4).

Since the permittivity of the dielectric layer is considered to exhibit Kerr type nonlinearity, the program used for the computational work calculates the value of nonlinear permittivity inside the dielectric layer by using expression given in (lb). In the transfer matrix approach the tangential components of the electric and magnetic fields across the interface are related as

$$
\left(\begin{array}{l}
E \\
H
\end{array}\right)_{z=0}=\left(\begin{array}{ll}
M_{11} & M_{12} \\
M_{21} & M_{22}
\end{array}\right)\left(\begin{array}{c}
E \\
H
\end{array}\right)_{z=d},
$$




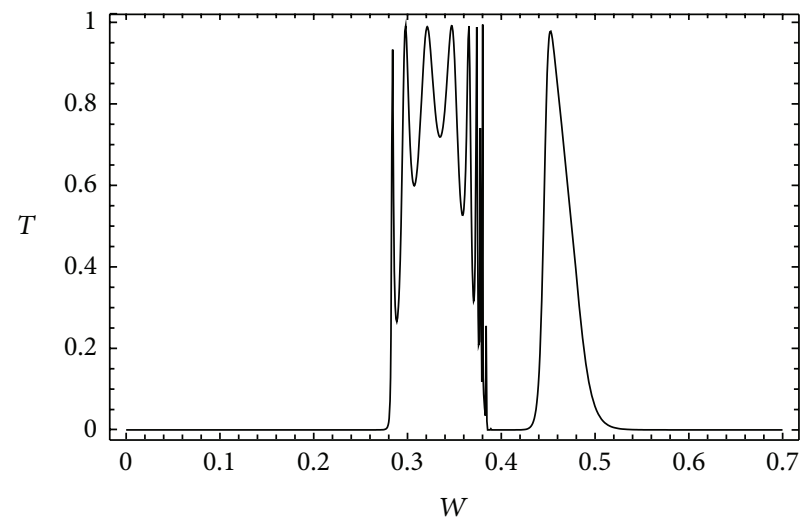

(a)



(b)

Figure 2: The transmission coefficient versus the frequency plots for (a) TM mode and (b) TE mode.

where

for TM mode,

$$
\begin{aligned}
& M_{11}=M_{22}=\cos \left(k_{z j} d_{j}\right), \\
& M_{12}=\frac{-\varepsilon_{j}}{k_{z j}} \sin \left(k_{z j} d_{j}\right), \\
& M_{21}=\frac{k_{z j}}{\varepsilon_{j}} \sin \left(k_{z j} d_{j}\right) ;
\end{aligned}
$$

for TE mode,

$$
\begin{aligned}
& M_{11}=M_{22}=\cos \left(k_{z j} d_{j}\right), \\
& M_{12}=\frac{-\mu_{j}}{k_{z j}} \sin \left(k_{z j} d_{j}\right), \\
& M_{21}=\frac{k_{z j}}{\mu_{j}} \sin \left(k_{z j} d_{j}\right) .
\end{aligned}
$$

In this approach, working through layer by layer the electric field at the transmitted end $E_{t}$ is calculated for an assumed incident electric field $E_{i}$ and the transmission coefficient of the structure is calculated by using the expression $T=$ $\left|E_{t}\right|^{2} /\left|E_{i}\right|^{2}$.

\section{Results and Discussions}

Initially we have taken $\alpha=0$ in (1b) and plotted the transmissivity versus frequency plot for both TM and TE modes for linear wave propagation in Figure 2. The periodic structure under consideration consists of 20 layers. As usual we are working here with dimensionless units, frequencies are normalized using $W=\omega d / c$, where $\omega$ is dimensional frequency, $d$ is the width of the period, and $c$ is the velocity of light. So the choice of working frequency depends on the dimensions of the structure under consideration. We have chosen $W_{\mathrm{pe}}=W_{\mathrm{pm}}=0.7$ and absorption is neglected in our analysis. Absorption only reduces transmission coefficient, so qualitatively same results are expected in the absorbing structures. From Figures 2(a) and 2(b), it is obvious that initially, for both TE and TM modes, there is no transmission across the structure, but, for certain frequency regions, the transmission coefficient becomes nearly unity. As these states decay exponentially away from the interface, within the layers, the modes are decaying modes. The existence of the propagating region can be explained by the tight binding model of the solid state physics [35]. The phenomenon is similar in nature to the origin of propagation modes in a periodic structure consisting of single negative material layers [31] with the difference that, in the present case, the fields get localized at each interface of the periodic structure along the $x$-axis.

For the nonlinear wave propagation, particular nonpropagating frequencies (i.e., 0.28 for the TM mode and 0.36 for the TE mode) near the edge of the propagating regions are chosen. The transmissivity versus the intensity curves at these particular frequencies for the nonlinear case are shown in Figures 3(a) and 3(b). The dimensionless Kerr coefficient $\alpha$ is taken to have the value of +1 . The phenomenon of transmission bistability is seen here but a comparison of part (a) and part (b) reveals the fact that although the electric and magnetic plasma frequencies are chosen to be the same, the structure shows sensitivity with respect to the polarization of the electromagnetic field. The horizontal scales of these two figures show that the intensity at which bistability phenomenon is observed for the TM mode is two orders of magnitude smaller than that value for the TE mode. Further it is observed that multiple bistability is observed for the TM mode whereas the phenomenon occurs only once for a considerable scale of intensity for the TE mode. The electric field profiles inside the structure at intensities corresponding to maximum transmission are shown in Figures 4(a) and 4(b). These profiles show that the light is tunneling through the structure to get out of it. For the TM mode, the profile corresponds to two solitons formation at the second transmission peak of the bistability curve and for the TE mode one soliton is formed at the single peak of the bistability curve. 


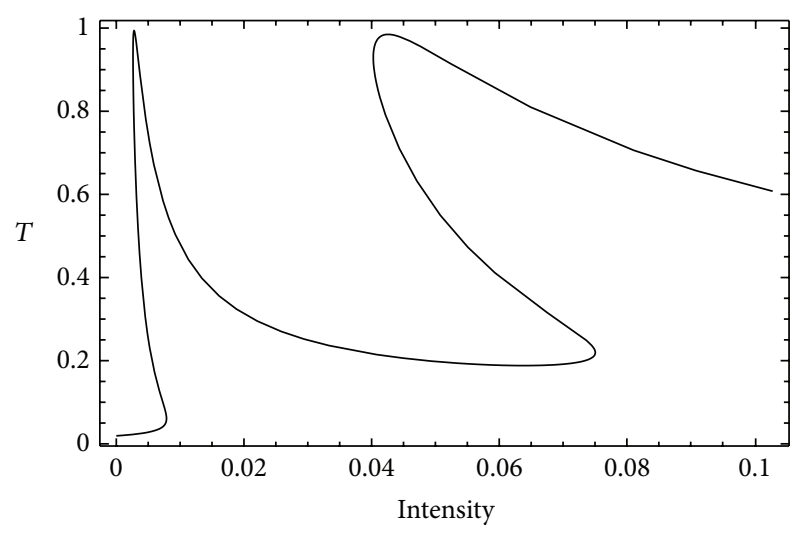

(a)

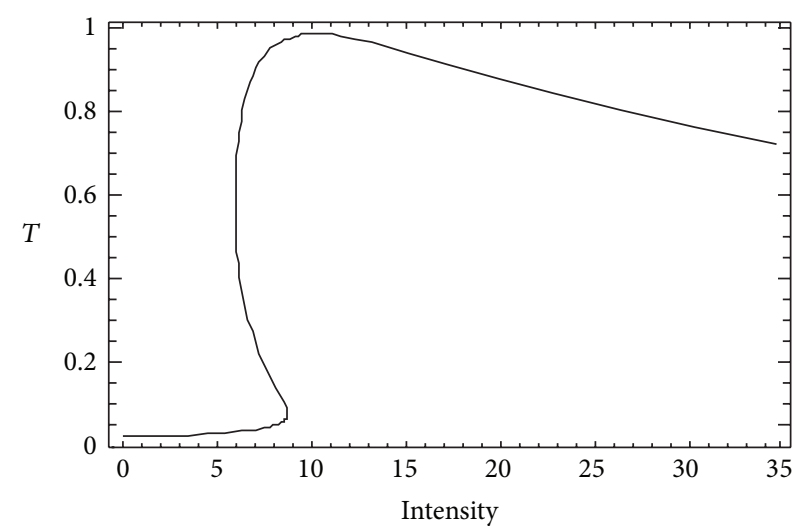

(b)

FIGURE 3: The transmission coefficient versus the intensity plot at (a) $W=0.28$ for the TM mode, (b) $W=0.36$ for the TE mode, and $\alpha=+1$ in both cases.

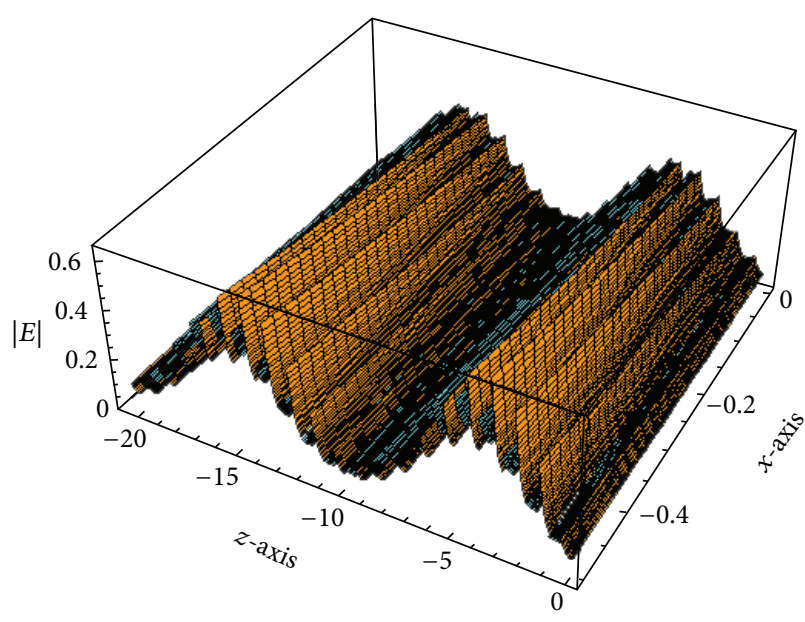

(a)



(b)

FIgURE 4: The electric field profiles inside the structure at (a) $W=0.28$ for the TM mode and (b) at $W=0.36$ for the TE mode.

\section{Conclusions}

In conclusion, we have studied tunneling of SPPs in onedimensional periodic structures composed of alternate lefthanded and dielectic (right-handed) layers. The local behavior of these states within each layer is quite opposite to that of the propagating states; that is, these states decay away from the interfaces but the transmission spectrum of these states resembles that for the propagating states; that is, we get propagating bands and nonpropagating gaps. For nonlinear wave propagation, the phenomenon of optical bistability is observed which corresponds to the formation of tunneling states inside the structures. These tunneling states provide a mean to transfer surface plasmon polariton along certain structures. It is obseved in these investigations that the structures show sensitivity with respect to the polarization of the electromagnetic field. The TM mode is more suitable for the observation of such phenomenon as compared to the TE mode. The phenomenon of nonlinear tunnelling of SPPs can be used in ultrafast nonlinear switches, nonlinear plasmonic sensors, surfaces enhanced Ramman scattering and waveguiding, and so forth [31]. It is hoped that this study can be useful in designing structures in which potential of these states is expolioted for nonlinear wave propagation.

\section{Conflict of Interests}

The author declares that there is no conflict of interests regarding the publication of this paper.

\section{References}

[1] R. H. Ritchie, E. T. Arakawa, J. J. Cowan, and R. N. Hamm, "Surface-plasmon resonance effect in grating diffraction," Physical Review Letters, vol. 21, no. 22, pp. 1530-1533, 1968.

[2] S. L. Cunningham, A. A. Maradudin, and R. F. Wallis, "Effect of a charge layer on the surface-plasmon-polariton dispersion curve," Physical Review B, vol. 10, no. 8, article 3342, 1974.

[3] M. Specht, J. D. Pedarnig, W. M. Heckl, and T. W. Hänsch, "Scanning plasmon near-field microscope," Physical Review Letters, vol. 68, no. 4, pp. 476-479, 1992. 
[4] W. L. Barnes, A. Dereux, and T. W. Ebbesen, "Surface plasmon subwavelength optics," Nature, vol. 424, no. 6950, pp. 824-830, 2003.

[5] A. V. Zayats, I. I. Smolyaninov, and A. A. Maradudin, "Nanooptics of surface plasmon polaritons," Physics Reports, vol. 408, no. 3-4, pp. 131-314, 2005.

[6] S. A. Maier, Plasmonics: Fundamentals and Applications, Springer Science+Business Media, 2007.

[7] S. Sidorenko and O. J. F. Martin, "Resonant tunneling of surface plasmon-polaritons," Optics Express, vol. 15, no. 10, pp. 63806388, 2007.

[8] L. Li, T. Li, S. M. Wang, and S. N. Zhu, "Collimated plasmon beam: nondiffracting versus linearly focused," Physical Review Letters, vol. 110, no. 4, Article ID 046807, 2013.

[9] H. H. Pham, I. Gourevich, J. K. Oh, J. E. N. Jonkman, and E. Kumacheva, "A multidye nanostructured material for optical data storage and security data encryption," Advanced Materials, vol. 16, no. 6, pp. 516-520, 2004.

[10] Y.-H. Chen, M. Q. Zhang, L. Gan et al., "Holographic plasmonic lenses for surface plasmons with complex wavefront profile," Optics Express, vol. 21, no. 15, pp. 17558-17566, 2013.

[11] M. Achermann, K. L. Shuford, G. C. Schatz, D. H. Dahanayaka, L. A. Bumm, and V. I. Klimov, "Near-field spectroscopy of surface plasmons in flat gold nanoparticles," Optics Letters, vol. 32, no. 15, pp. 2254-2256, 2007.

[12] G. A. Wurtz, R. Pollard, and A. V. Zayats, "Optical bistability in nonlinear surface-plasmon polaritonic crystals," Physical Review Letters, vol. 97, no. 5, Article ID 057402, 2006.

[13] F. Tao, H.-F. Zhang, X.-H. Yang, and D. Cao, "Surface plasmon polaritons of the metamaterial four-layered structures," Journal of the Optical Society of America B, vol. 26, no. 1, pp. 50-59, 2009.

[14] R. Ruppin, "Surface polaritons of a left-handed material slab," Journal of Physics: Condensed Matter, vol. 13, pp. 1811-1819, 2001.

[15] H.-F. Zhang, D. Cao, F. Tao et al., "Surface plasmon polaritons of symmetric and asymmetric metamaterial slabs," Chinese Physics B, vol. 19, no. 2, Article ID 027301, 2010.

[16] I. V. Shadrivov, A. A. Sukhorukov, Y. S. Kivshar, A. A. Zharov, A. D. Boardman, and P. Egan, "Nonlinear surface waves in lefthanded materials," Physical Review E, vol. 69, no. 1, Article ID 016617, 2004.

[17] M. S. Hamada, A. H. El-Astal, M. A. Sbiah, and M. M. Shabat, "Analytical approach of the nonlinear surface plasmon at a lefthanded material," Journal of Applied Mathematics and Physics, vol. 3, no. 6, pp. 673-679, 2015.

[18] V. G. Veselago, "The electrodynamics of substances with simultaneously negative values of $\varepsilon$ AND $\mu$," Soviet Physics Uspekhi, vol. 10, no. 4, pp. 509-514, 1968.

[19] N. Engheta and R. W. Ziolkowski, Metamaterials: Physics and Engineering Explorations, John Wiley \& Sons, 2006.

[20] J. B. Pendry, A. J. Holden, W. J. Stewart, and I. Youngs, "Extremely low frequency plasmons in metallic mesostructures," Physical Review Letters, vol. 76, no. 25, pp. 4773-4776, 1996.

[21] J. B. Pendry, A. J. Holden, D. J. Robbins, and W. J. Stewart, "Magnetism from conductors and enhanced nonlinear phenomena," IEEE Transactions on Microwave Theory and Techniques, vol. 47, no. 11, pp. 2075-2084, 1999.

[22] D. R. Smith, D. C. Vier, N. Kroll, and S. Schultz, "Direct calculation of permeability and permittivity for a left-handed metamaterial," Applied Physics Letters, vol. 77, no. 14, pp. 22462248, 2000.
[23] J.-F. Wang, S.-B. Qu, Z. Xu et al., "Experimental verification of left-handed metamaterials composed of coplanar electric and magnetic resonators," Chinese Physics Letters, vol. 27, no. 3, Article ID 034104, 2010.

[24] A. Alu and N. Engheta, "Optical nanotransmission lines: synthesis of planar left-handed metamaterials in the infrared and visible regimes," Journal of the Optical Society of America B, vol. 23, no. 3, pp. 571-583, 2006.

[25] G. Torosyan, C. Rau, B. Pradarutti, and R. Beigang, "Generation and propagation of surface plasmons in periodic metallic structures," Applied Physics Letters, vol. 85, no. 16, article 3372, 2004.

[26] N. C. Panoin and R. M. Osgood Jr., "Linear and nonlinear transmission of surface plasmon polaritons in an optical nanowire," in Organic and Nanocomposite Optical Materials, vol. 846 of MRS Symposium Proceedings, pp. DD5.6.1-DD5.6.6, Materials Research Society, Warrendale, Pa, USA, 2004.

[27] Y.-G. Chen, Y.-H. Chen, and Z.-Y. Li, "Direct method to control surface plasmon polaritons on metal surfaces," Optics Letters, vol. 39, no. 2, pp. 339-342, 2014.

[28] S. M. Wang and L. Gao, "Nonlinear responses of the periodic structure composed of single negative materials," Optics Communications, vol. 267, no. 1, pp. 197-204, 2006.

[29] S. M. Wang, C. J. Tang, T. Pan, and L. Gao, "Bistability and gap soliton in one-dimensional photonic crystal containing singlenegative materials," Physics Letters A, vol. 348, no. 3-6, pp. 424431, 2006.

[30] M. Kauranen and A. V. Zayats, "Nonlinear plasmonics," Nature Photonics, vol. 6, no. 11, pp. 737-748, 2012.

[31] Y.S. Kivshar, "From metamaterials to metasurfaces and metadevices," Nanosystems: Physics, Chemistry, Mathematics, vol. 6, no. 3, pp. 346-352, 2015.

[32] M. Z. Ali and T. Abdullah, "Properties of the angular gap in a one-dimensional photonic band gap structure containing single negative materials," Physics Letters A, vol. 372, no. 10, pp. 16951700, 2008.

[33] P. Markos and C. M. Soukoulis, Wave Propagation from Electrons to Photonic Crystals and Left-Handed Materials, Princeton University Press, Princeton, NJ, USA, 2008.

[34] C. Wu, G. Song, H. Liu, L. Cui, L. Yu, and J. Xiao, "Optical bistability of surface plasmon polaritons in nonlinear Kretschmann configuration," Journal of Modern Optics, vol. 60, no. 3, pp. 190196, 2013.

[35] N. W. Ashcroft and N. D. Mermin, Solid State Physics, Thomson Learning, Toronto, Canada, 1976. 

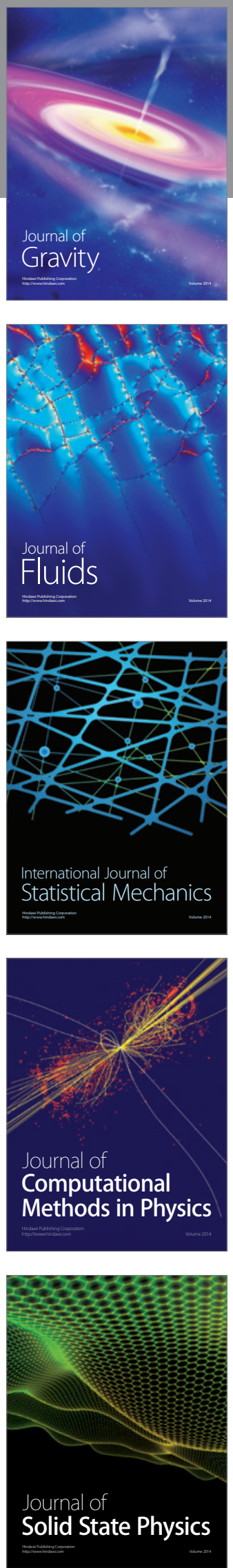

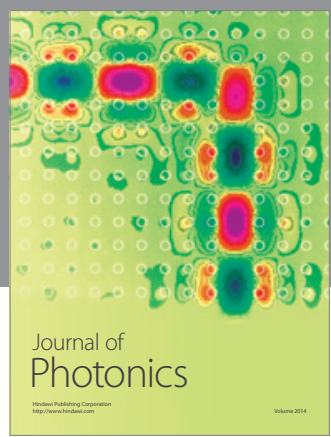

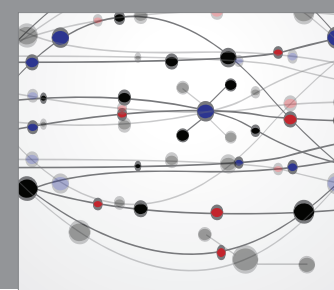

The Scientific World Journal

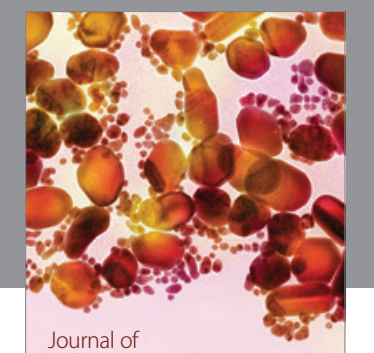

Soft Matter
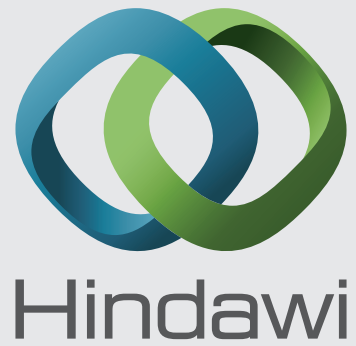

Submit your manuscripts at

http://www.hindawi.com
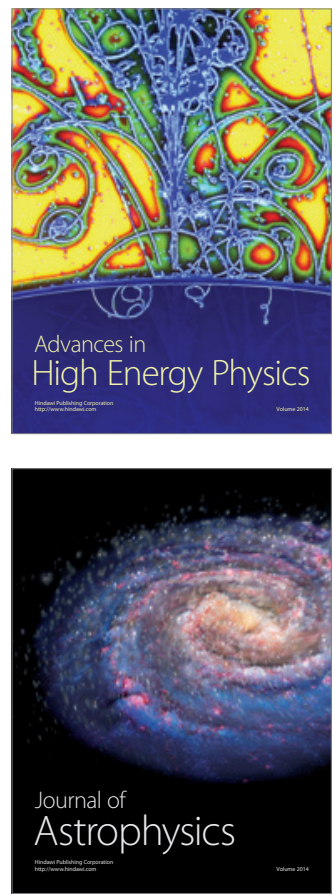
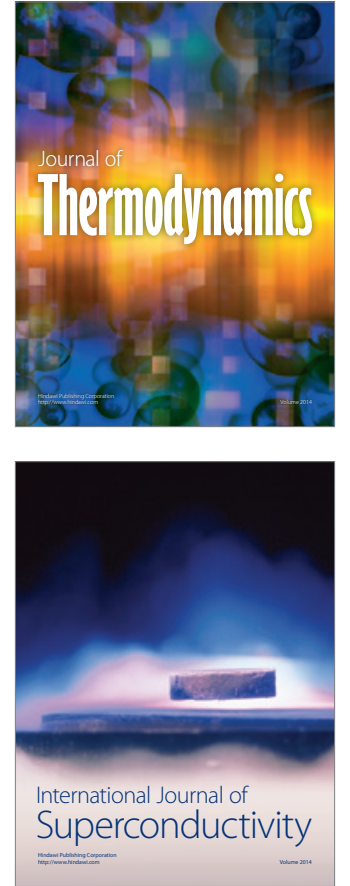
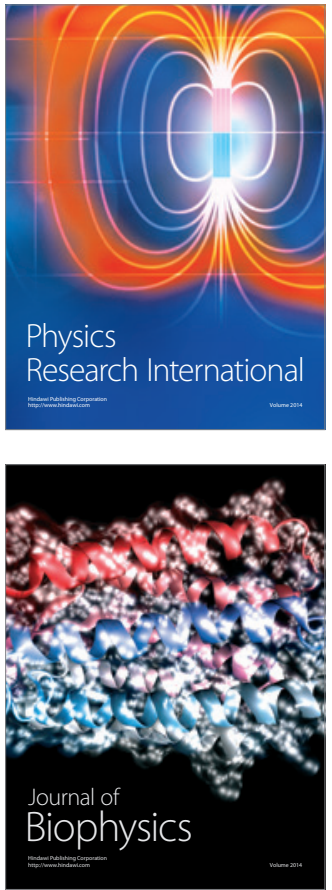
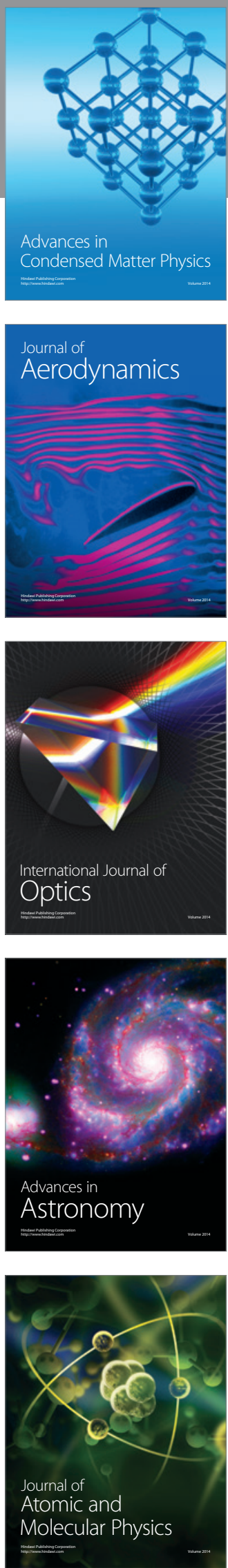